\title{
Positive Solutions for Nonlinear Integro-Differential Equations of Mixed Type in Banach Spaces
}

\author{
Yan Sun ${ }^{1,2}$ \\ ${ }^{1}$ School of Mathematical Sciences, Fudan University, Shanghai 200433, China \\ ${ }^{2}$ Department of Mathematics, Shanghai Normal University, Shanghai 200234, China
}

Correspondence should be addressed to Yan Sun; ysun@shnu.edu.cn

Received 29 August 2013; Accepted 16 September 2013

Academic Editor: Geraldo Botelho

Copyright (C) 2013 Yan Sun. This is an open access article distributed under the Creative Commons Attribution License, which permits unrestricted use, distribution, and reproduction in any medium, provided the original work is properly cited.

We establish some new existence theorems on the positive solutions for nonlinear integro-differential equations which do not possess any monotone properties in ordered Banach spaces by means of Banach contraction mapping principle and cone theory based on some new comparison results.

\section{Introduction}

In this paper, we consider the existence of the unique positive solution and at least one positive solution for the following initial value problem (IVP) of the nonlinear integrodifferential equation of mixed type in ordered Banach spaces E:

$$
\begin{gathered}
u^{\prime \prime}(t)=g(t, u(t),(T u)(t),(S u)(t)), \quad \forall t \in I, \\
u(0)=u_{0}, \quad u^{\prime}(0)=u_{1},
\end{gathered}
$$

where $I=[0, a](a>0), g(t, u(t),(T u)(t),(S u)(t))=f(t$, $u(t), u(t),(T u)(t),(S u)(t)), u_{0}, u_{1} \in E, f \in C[I \times P \times P \times P \times$ $P, P]$, and $f$ is nonincreasing with the second variable $u(t)$, with $f$ is nondecreasing with the third variable $u(t) . P$ is a positive cone in ordered Banach spaces $E$, and

$$
\begin{gathered}
(T u)(t)=\int_{0}^{t} k(t, s) u(s) d s, \\
(S u)(t)=\int_{0}^{a} h(t, s) u(s) d s, \quad t \in I .
\end{gathered}
$$

In (2), $k \in C\left[D, R^{+}\right]$and $h \in C\left[D_{0}, R^{+}\right]$, where $D=\{(t, s) \in$ $R \times R: 0 \leq s \leq t \leq a\}, D_{0}=\{(t, s) \in R \times R:(t, s) \in I \times I\}, R^{+}$ denotes the set of nonnegative real numbers, and $R$ denotes the set of real numbers. In the special case when $f$ does not contain the third argument, Guo [1] proved the minimal and maximal solution of the following initial value problem:

$$
u^{\prime}=f(t, u, T u), \quad t \in I, \quad u\left(t_{0}\right)=x_{0},
$$

under some stronger conditions. He used the topological degree theory and the monotone iterative technique. When $f$ does not possess any monotone assumption, the problem of proving the existence results is an interesting and important question. The aim of the paper is to study this kind of problem. By means of Banach contraction mapping principle and cone theory based on some new comparison results, we obtain some new existence theorems of the solutions for the initial value problems of the nonlinear integro-differential equations of mixed type which does not possess any monotone properties in ordered Banach spaces. The new results obtained are quite general and are used to generalize, improve, and unify many recent results of [2-8]. Their results assert the existence of one solution, the minimal and maximal solution using monotone iterative technique with the stronger conditions. Our method is different from the method of mixed monotony, and the results obtained in the paper are new even if the space $E$ is finite dimensional.

Inspired and motivated greatly by the above work, the aim of the paper is to consider the existence of positive solutions for the boundary value problem (1) under simpler conditions. The main results of problem (1) are obtained by making use 
of new fixed point theorem. More specifically, in the proof of these theorems, we establish new comparison theorem and construct a special cone for strict set contraction operator. Our main results in essence improve and generalize the corresponding results of $[1,9-12]$. Moreover, our method is different from those in $[5,10,13]$.

The rest of the paper is organized as follows. In Section 2, we present some known results and introduce conditions to be used in the next section. The main theorems are formulated and proved in Section 3.

\section{Preliminaries and Lemmas}

In this section, we will state some necessary definitions and preliminary results.

Definition 1. Let $E$ be a real Banach space. A nonempty closed set $P \subset E$ is called a cone if it satisfies the following two conditions:

(1) $z \in P, \lambda>0$ implies $\lambda z \in P$;

(2) $z \in P,-z \in P$ implies $z=\theta$, where $\theta$ denotes the zero element of $E$.

A cone is said to be solid if it contains interior points, $\stackrel{\circ}{P} \neq \theta$. The positive cone $P$ is said to be generating if $E=P-P$; that is, every element $y \in E$ can be represented in the form $y=x-z$, where $x, z \in P$. Every cone with nonempty interior is generating. A cone $P$ induces a partial ordering in $E$ given by $u \leq v$ if $v-u \in P$. If $u \leq v$ and $u \neq v$, we write $u<v$; if cone $P$ is solid and $v-u \in \stackrel{\circ}{P}$, we write $u \ll v$.

Definition 2. A cone $P \subset E$ is said to be normal if there exists a positive constant $v$ such that $\|x+y\| \geq v, \forall x, y \in P,\|x\|=1$, $\|y\|=1$.

Proposition 3. A positive cone $P$ is called normal if and only if there exists $N>0$ such that $0 \leq x \leq y$ implies $\|x\| \leq N\|y\|$. The norm on $E$ is called monotone if $0 \leq u \leq v$ implies $\|u\| \leq$ $\|v\|$. The cone $P$ is said to be normal if and only if there exists an equivalent monotone norm on $E$.

For further details on cone theory, one can refer to $[2,14]$.

Let $P=\{u \in C[I, E]: u(t) \geq \theta$ for $t \in I\}$, where $C[I, E]$ denotes the Banach space of all continuous mapping $u: I \rightarrow E$ with norm $\|u\|=\max \{u: t \in I\}$. It is easy to see that $P$ is a cone in $C[I, E]$, and so it defines a partial order in $C[I, E]$ by $u \leq v$ if and only if $u(t) \leq v(t)$ for all $t \in I$. Given a cone $P$, the dual of $P$ is defined as $P^{*}=\left\{\varphi \in E^{*}: \varphi(u) \geq 0\right.$ for $\left.u \in P\right\}$, where $E^{*}$ is the dual of $E$.

Let $k_{0}=\max \{k(t, s):(t, s) \in D\}, h_{0}=\max \{h(t, s):$ $\left.(t, s) \in D_{0}\right\}$. For any $H \subset C[I, E]$ and $t \in I$, let

$$
\begin{aligned}
H(t) & =\{u(t): u \in H\}, \\
(T H)(t) & =\{(T u)(t): u \in H\}, \\
(S H)(t) & =\{(S u)(t): u \in H\} .
\end{aligned}
$$

Definition 4. Let $E$ be a metric space and $S$ be a bounded subset of $E$. The measure of noncompactness $\Upsilon(S)$ of $S$ is defined by

$$
\begin{gathered}
\Upsilon(S)=\inf \{\delta>0: S \text { admits a finite cover by sets } \\
\text { of diameter } \leq \delta\} .
\end{gathered}
$$

Definition 5. An operator $B: D \rightarrow E$ is said to be completely continuous if it is continuous and compact. $B$ is called a $k$ set-contraction $(k \geq 0)$ if it is continuous, bounded, and $\Upsilon(B(S)) \leq k \Upsilon(S)$ for any bounded set $S \subset D$, where $\Upsilon(S)$ denotes the measure of noncompactness of $S$.

A $k$-set-contraction is called a strict-set-contraction if $k<1$. An operator $B$ is said to be condensing if it is continuous, bounded, and $\Upsilon(B(S))<\Upsilon(S)$ for any bounded set $S \subset D$ with $\Upsilon(S)>0$.

Obviously, if $B$ is a strict-set-contraction, then $B$ is a condensing mapping, and if operator $B$ is completely continuous, then $B$ is a strict-set-contraction.

Definition 6 (see [15]). Let $E$ be a Banach space, and let $A$ : $E \times E \rightarrow E$. The mapping $A$ is said to have the mixed monotone property if $A(x, y)$ is monotone nonincreasing in $u$ and is monotone nondecreasing in $v$; that is,

$$
\begin{array}{ll}
\text { for any } u_{1}, u_{2} \in E, & u_{1} \leq u_{2} \Longrightarrow A\left(u_{1}, v\right) \geq A\left(u_{2}, v\right), \\
\text { for any } v_{1}, v_{2} \in E, & v_{1} \leq u_{2} \Longrightarrow A\left(u, v_{1}\right) \leq A\left(u, v_{2}\right) .
\end{array}
$$

Definition 7 (see [15]). A couple point $(x, y) \in E \times E$ is said to be a coupled fixed point of the mapping $A: E \times E \rightarrow E$ if $u=A(u, v)$ and $v=A(v, u)$.

Lemma 8 (see $[2,14])$. A positive cone $P$ is normal and generating if and only if there exists a positive constant $\tau>0$ and $y, z \in P$ such that $x=y-z$ for all $x \in P$ and $\|y\| \leq \tau\|x\|,\|z\| \leq \tau\|x\|$.

The following lemma plays a key role for improving the main results.

Lemma 9. Let $P$ be a normal generate cone, and let $A$ : $P \times P \rightarrow P$ be a nonlinear operator. If there exists a positive bounded linear operator $L: P \rightarrow P$ such that $r(L)<1$ and

$$
\begin{aligned}
-L(x-\bar{x}+\bar{y}-y) & \leq A(x, y)-A(\bar{x}, \bar{y}) \\
& \leq L(x-\bar{x}+\bar{y}-y), \\
\forall x, \bar{x}, y, \bar{y} & \in P, x \geq \bar{x}, \quad y \leq \bar{y},
\end{aligned}
$$

then $A$ has a unique positive fixed point $x^{*}$, and for any $x_{0} \in P$, let $x_{n}=A\left(x_{n-1}, x_{n-1}\right) \quad(n=1,2, \ldots), x_{n} \rightarrow x^{*} \quad(n \rightarrow \infty)$.

Proof. Since $r(L)<1$, then $r(L)=\lim _{n \rightarrow \infty}\left\|L^{n}\right\|^{1 / n}<1$. Thus, there exists a natural number $n_{0}$ and $0<\alpha<1$ such that

$$
\left\|L^{n_{0}}\right\| \leq \alpha^{n_{0}}
$$


Since $P$ is generate, it follows from Lemma 8 that there exist $\tau>0, y, z \in P$ such that $x=y-z$ and

$$
\|y\| \leq \tau\|x\|, \quad\|z\| \leq \tau\|x\|, \quad \forall x \in P .
$$

Then $\|y+z\| \leq 2 \tau\|x\|, \forall x \in P$. Suppose that $x, y, z \in P$ are not in the same line for any $0 \leq x-y \leq z$. Let $u_{1}=(1 / 2)(x+$ $y-z), u_{2}=x-y+z, u_{3}=-x+y+z$. Then $u_{1}, u_{2}, u_{3} \in P$ and $x \geq y \geq u_{1}, x-u_{1}=(1 / 2) u_{2}, y-u_{1}=(1 / 2) u_{3}, u_{2}+u_{3}=2 z$. It follows from (7) that

$$
\begin{gathered}
-\frac{L}{2} u_{2} \leq A(x, x)-A\left(u_{1}, x\right) \leq \frac{L}{2} u_{2}, \\
-\frac{L}{2}\left(u_{2}+u_{3}\right) \leq A\left(u_{1}, x\right)-A\left(y, u_{1}\right) \\
\leq \frac{L}{2}\left(u_{2}+u_{3}\right), \\
-\frac{L}{2} u_{3} \leq A\left(y, u_{1}\right)-A(y, y) \leq \frac{L}{2} u_{3} .
\end{gathered}
$$

By virtue of $(10)+(12)-(11)$, we have

$$
\begin{gathered}
-\frac{L}{2}\left(u_{2}+u_{3}\right) \leq A(x, x)-A(y, y) \leq \frac{L}{2}\left(u_{2}+u_{3}\right), \\
-L \cdot 2 z \leq A(x, x)-A(y, y) \leq L \cdot 2 z .
\end{gathered}
$$

We first define the operator $\widetilde{A}: P \rightarrow P$ as the following $\widetilde{A} x=$ $A(x, x), x \in P$. Let $w=2 z$. Then $-L w \leq \widetilde{A} x-\widetilde{A} y \leq L w$. Since $L$ is a positive bounded linear operator, then $L u \in P$. By induction, it is easy to see that

$$
-L^{n} w \leq \widetilde{A}^{n} x-\widetilde{A}^{n} y \leq L^{n} w, \quad L^{n} w \in P .
$$

Since $w \in P$ is arbitrary and $n_{0}$ is taken as in (8), we get

$$
\left\|\widetilde{A}^{n_{0}} x-\widetilde{A}^{n_{0}} y\right\| \leq L^{n_{0}}\|x-y\| \leq \alpha^{n_{0}}\|x-y\| .
$$

From $0<\alpha<1$ and Banach contract principle, we know that $\widetilde{A}^{n_{0}}$ has the unique positive fixed point $x^{*}$ in $P$; that is, $x^{*}$ is the unique positive solution of operator equation $x=A(x, x)$. And for any $x_{0} \in E$, let $x_{n}=A\left(x_{n-1}, x_{n-1}\right)(n=1,2, \ldots)$, we have $\left\|x_{n}-x^{*}\right\| \rightarrow 0(n \rightarrow \infty)$.

Remark 10. The results of Lemma 9 obtained are quite general and are used to improve, generalize, and unify many results of Chen [13], Guo [9], Krasnosel'skii and Zabreiko [16], Zhang [11], Su et al. [10], and Liu [12]. Not only do we obtain the existence and uniqueness of fixed points for mixed nonmonotone binary operators in ordered Banach spaces, but we also solve the difficult open problem that nonmonotone binary operator has unique fixed points under some weaker conditions.

Lemma 11 (see [17]). Let E be a Banach space and $H \subset C[I, E]$ if $H$ is a countable set of strongly measurable functions $y$ : $I \rightarrow$ E such that there exists $\varphi \in L\left[I, R^{+}\right]$such that $\|y(t)\| \leq$ $\varphi(t), t \in I, y \in H$. Then $\Upsilon(H(t))$ is Lebesgue integrable on $I$, and

$$
\Upsilon\left(\left\{\int_{I} y(s) d s: y \in H\right\}\right) \leq 2 \int_{I} \Upsilon(H(s)) d s
$$

Lemma 12 (see $[1,18]$ ). Let $H \subset C[J, E]$ be bounded and equicontinuous; then $\Upsilon(H(t))$ is continuous on $I$, and

$$
\Upsilon\left(\int_{I} H(s) d s\right) \leq \int_{I} \Upsilon(H(s)) d s .
$$

Lemma 13 (see $[2,19])$. Let $X$ be a Banach space, $K \subset X$ closed and convex, and $F: K \rightarrow K$ continuous with the further property that for some $x \in K$ we have $B \subset K$ countable. $\bar{B}=$ $\overline{\mathrm{co}}(\{\alpha\} \cap F(B))$ implies that $B$ is relatively compact. Then $F$ has a fixed point in $K$.

Lemma 14. Assume that $m \in C\left[I, R^{+}\right]$satisfies

$$
m(t) \leq M \int_{t_{0}}^{t} m(s) d s+M^{*} \int_{t_{0}}^{t+a} m(s) d s, \quad t \in I,
$$

where $M>0$ and $M^{*} \geq 0$ are constants. Then $m(t) \equiv 0$ for $t \in I$ provided one of the following two conditions hold:

(i) $M>M^{*}\left(e^{M\left(t-t_{0}\right)}-1\right)$;

(ii) $a\left(M+M^{*}\right)<1$.

Proof. Suppose that (i) holds. Let $p(t)=\int_{t_{0}}^{t} m(s) d s$; then $p\left(t_{0}\right)=0, p^{\prime}(t)=m(t) \geq 0$, for $t \in I$. From (18), we know that

$$
p^{\prime}(t) \leq M p(t)+M^{*} p\left(t_{0}+a\right), \quad t \in I .
$$

Since $e^{-M\left(t-t_{0}\right)}>0$, we have

$$
\left(p(t) e^{-M\left(t-t_{0}\right)}\right)^{\prime} \leq M^{*} p\left(t_{0}+a\right) e^{-M\left(t-t_{0}\right)}, \quad t \in I .
$$

Integrate from $t_{0}$ to $t$; noticing $p\left(t_{0}\right)=0$, we have

$$
\begin{aligned}
0 & \leq p(t) e^{-M\left(t-t_{0}\right)} \leq \int_{t_{0}}^{t} M^{*} p\left(t_{0}+a\right) e^{-M\left(s-t_{0}\right)} d s \\
& =\frac{M^{*}}{M} p\left(t_{0}+a\right)\left(1-e^{M\left(t-t_{0}\right)}\right), \quad t \in I .
\end{aligned}
$$

Hence,

$$
0 \leq p(t) \leq \frac{M^{*}}{M} p\left(t_{0}+a\right)\left(e^{M\left(t-t_{0}\right)}-1\right), \quad t \in I .
$$

We claim that

$$
p\left(t_{0}+a\right)=0 \text {. }
$$

Otherwise, $p\left(t_{0}+a\right)>0$; then we take $t=t_{0}+a$ in (22), we obtain $M \leq M^{*}\left(e^{M\left(t-t_{0}\right)}-1\right)$, which contradicts assumption (i).

Therefore, from (22) and (23), we know that $p(t)=$ $\int_{t_{0}}^{t} m(s) d s=0$. Consequently, $m(s) \equiv 0$ a.e. on $t \in I$.

Let us suppose now that (ii) holds. Then

$$
m(t) \leq\left(M+M^{*}\right) \int_{t_{0}}^{t_{0}+a} m(s) d s, \quad t \in I .
$$


It follows by integrating the above inequality that

$$
\int_{t_{0}}^{t_{0}+a} m(s) d s \leq a\left(M+M^{*}\right) \int_{t_{0}}^{t_{0}+a} m(s) d s,
$$

which, by assumption (ii), implies that $\int_{t_{0}}^{t_{0}+a} m(s) d s=0$ and so $m(t) \equiv 0$ a.e. on $I$. The proof of Lemma 14 is therefore complete.

Lemma 15 (see $[2,19]) . H \subset C^{1}[I, E]$ is relatively compact if and only if each element $y(t) \in H$ and $y^{\prime}(t) \in H$ are uniformly bounded and equicontinuous on $I$.

\section{Main Results}

We are now in a position to prove our main results concerning the unique positive solution and at least one positive solution.

Theorem 16. Suppose that $P$ is a normal solid cone whenever $t \in I$; there exists $\widetilde{M}>0, y_{1}, \bar{y}_{1}, y_{2}, \bar{y}_{2} \in P, y_{1} \geq \bar{y}_{1}, y_{2} \leq \bar{y}_{2}$ such that

$$
\begin{array}{r}
f\left(t, y_{1}, y_{2}, T y_{1}, S y_{1}\right)-f\left(t, \bar{y}_{1}, \bar{y}_{2}, T \bar{y}_{1}, S \bar{y}_{1}\right) \\
\geq-\widetilde{M}\left[y_{1}-\bar{y}_{1}+\bar{y}_{2}-y_{2}+T y_{1}-T \bar{y}_{1}\right], \\
f\left(t, y_{1}, y_{2}, T y_{1}, S y_{1}\right)-f\left(t, \bar{y}_{1}, \bar{y}_{2}, T \bar{y}_{1}, S \bar{y}_{1}\right) \\
\leq \widetilde{M}\left[y_{1}-\bar{y}_{1}+\bar{y}_{2}-y_{2}+T y_{1}-T \bar{y}_{1}\right] .
\end{array}
$$

Then IVP (1) has the unique positive solution $x^{*}$ in $C^{2}[I, P]$.

Proof. Define operator $A: C[I, P] \times C[I, P] \rightarrow C[I, P]$ as the following:

$$
\begin{aligned}
A(u, v)= & u_{0}+t u_{1} \\
& +\int_{0}^{t}(t-s) f(s, u(s), v(s), \\
& (\mathrm{Tu})(s),(\mathrm{Su})(s)) d s, \quad t \in I .
\end{aligned}
$$

Then $u \in C^{2}[I, P]$ is a solution of IVP (1) if and only if $u \in C[I, P]$ is a fixed point of $A$; that is, $u=A(u, u)$ for any $u, v, \bar{u}, \bar{v} \in C[I, P], u \geq \bar{u}, v \leq \bar{v}$. From (26) and (28), we know that

$$
\begin{aligned}
A(u, v) & -A(\bar{u}, \bar{v}) \\
& \geq-\widetilde{M} a \int_{0}^{t}[(u-\bar{u})+(\bar{v}-v)+T(u-\bar{u})] d s \\
& \geq-\widetilde{M} a\left[(1+a) \int_{0}^{t}(u-\bar{u}) d s+\int_{0}^{t}(\bar{v}-v) d s\right] \\
& \geq-\widetilde{M} a(1+a) \int_{0}^{t}(u-\bar{u}+\bar{v}-v) d s,
\end{aligned}
$$

$$
\begin{aligned}
& A(u, v)-A(\bar{u}, \bar{v}) \\
& \leq \widetilde{M} a \int_{0}^{t}[(u-\bar{u})+(\bar{v}-v)+T(u-\bar{u})] d s \\
& \leq \widetilde{M} a\left[(1+a) \int_{0}^{t}(u-\bar{u}) d s+\int_{0}^{t}(\bar{v}-v) d s\right] \\
& \leq \widetilde{M} a(1+a) \int_{0}^{t}(u-\bar{u}+\bar{v}-v) d s .
\end{aligned}
$$

From (29) and (30), for any $u, v, \bar{u}, \bar{v} \in C[I, P], u \geq \bar{u}, v \leq \bar{v}$ we know that

$$
-L(u-\bar{u}+\bar{v}-v) \leq A(u, v)-A(\bar{u}, \bar{v}) \leq L(u-\bar{u}+\bar{v}-v),
$$

where

$$
(L w)(t)=\widetilde{M} a(1+a) \int_{0}^{t} w(s) d s .
$$

Now we prove that $r(L)<1$, for $t \in I$. From (32), we know that

$$
\|(L u)(t)\|=\widetilde{M} a(1+a) t\|u\| .
$$

Denote $\eta=\widetilde{M} a(1+a)$. From (32) and (33), we get

$$
\begin{aligned}
\left\|\left(L^{2} u\right)(t)\right\| & \leq \eta \int_{0}^{t}(L u)(s) d s \\
& \leq \eta\|u\| \int_{0}^{t} s d s=\frac{\eta^{2} t^{2}}{2 !}\|u\|, \quad \forall t \in I .
\end{aligned}
$$

By induction, for any natural number $n$, we have

$$
\begin{aligned}
& \left\|\left(L^{n} u\right)(t)\right\| \leq \frac{\eta^{n} t^{n}}{n !}\|u\|, \quad \forall t \in I, \\
& \left\|L^{n} u\right\|=\max _{t \in I}\left\|\left(L^{n} u\right)(t)\right\| \leq \frac{\eta^{n} t^{n}}{n !}\|u\| .
\end{aligned}
$$

Therefore, $r(L)=\lim _{n \rightarrow \infty}\left\|L^{n}\right\|^{1 / n}=0<1$.

Since $P$ is normal solid cone in $C[I, E]$ and $P$ is generate, by virtue of Lemma 9 , we know that $A$ has a unique positive fixed point $x^{*}$ in $P$; that is, IVP (1) has a unique positive solution $x^{*}$ in $C^{2}[I, P] \subset C^{2}[I, E]$.

Remark 17. The conditions of Theorem 16 cannot be obtained by Chen and Zhuang [3], Lakshmikantham et al. [8], and Sun and Liu [20]. We obtain a unique positive solution of IVP (1). The conditions imposed on nonlinear term $f$ are sharper, and the result is new.

Denote $M^{\prime}(R)=\sup \{\|f(t, u, v, w, z)\|:(t, u, v, w, z) \in$ $\left.I \times B_{R} \times B_{R} \times B_{R} \times B_{R}\right\}, B_{R}=\{x \in P:\|x\| \leq R\}$.

Theorem 18. Assume that $f: I \times P \times P \times P \times P \rightarrow P$ satisfying the following conditions.

$\left(\mathrm{H}_{1}\right)$ For any $R>0, f$ is uniformly continuous on $I \times B_{R} \times$ $B_{R} \times B_{R} \times B_{R}$ and

$\varlimsup_{R \rightarrow \infty} \frac{M^{\prime}(R)}{R}<\left(a a_{0}\right)^{-1}, \quad$ where $a_{0}=\max \left\{1, a k_{0}, a h_{0}\right\}$. 
$\left(\mathrm{H}_{2}\right)$ There exists $L>0$ such that

$$
\begin{aligned}
& \Upsilon(f(t, B(t), B(t),(T B)(t),(S B)(t))) \\
& \quad \leq \lambda_{1} \Upsilon(B(t))+\lambda_{2} \Upsilon((T B)(t))+\lambda_{3} \Upsilon((S B)(t))
\end{aligned}
$$

for any bounded set $S \subset C[I, P] \subset C[I, E], t \in I$, and $2\left(\lambda_{1}+\right.$ $\left.\lambda_{2} a k_{0}\right)>0,2 \lambda_{3} h_{0} a \geq 0$ with $2 a\left(\lambda_{1}+\lambda_{2} a k_{0}+\lambda_{3} a h_{0}\right)<1$.

Then IVP (1) has at least one positive solution in $C^{2}[I, P] \subset$ $C^{2}[I, E]$.

Proof. We first define the operator $F: C[I, P] \rightarrow C[I, P]$ by the formula

$(F u)(t)=u_{0}+t u_{1}$

$$
+\int_{0}^{t}(t-s) f(s, u(s), u(s),(T u)(s),(S u)(s)) d s,
$$

It is easy to know that $u$ is a solution of IVP (1) if and only if $u$ is a fixed point of $F$.

It follows from $\left(\mathrm{H}_{1}\right)$ that there exist $0<r<\left(a a_{0}\right)^{-1}$ and $R_{1}>0$ such that

$$
\frac{M^{\prime}(R)}{R}<r, \quad \text { for } R \geq a_{0} R_{1} .
$$

Let

$$
\begin{aligned}
& R^{*}=\max \left\{R_{1},\left(\left\|u_{0}\right\|+a\left\|u_{1}\right\|\right)\left(1-a a_{0} r\right)^{-1}\right\}, \\
& B_{I}\left(R^{*}\right)=\left\{u \in C[I, P]:\|u\| \leq R^{*}\right\} .
\end{aligned}
$$

Then for any $u \in B_{I}\left(R^{*}\right)$, we have

$$
\begin{aligned}
& \|u\|=R^{*} \leq a_{0} R^{*}, \quad\|T u\| \leq a k_{0}\|u\| \leq a k_{0} R^{*} \leq a_{0} R^{*}, \\
& \|S u\| \leq a h_{0}\|u\| \leq a h_{0} R^{*} \leq a_{0} R^{*} .
\end{aligned}
$$

It follows from (38) and (39) that

$$
\begin{aligned}
\|F u\| & \leq\left\|u_{0}\right\|+a\left\|u_{1}\right\|+a M^{\prime}\left(a_{0} R^{*}\right) \\
& \leq\left\|u_{0}\right\|+a\left\|u_{1}\right\|+a a_{0} R^{*} r \leq R^{*} .
\end{aligned}
$$

Set $K=\overline{\mathrm{CO}}\left(B_{I}\left(R^{*}\right)\right)$. Then $F$ is a continuous operator from $K$ into $K$. It is easy to see from $(38),\left(\mathrm{H}_{1}\right)$, and the normality of $P$ that $K \subset B_{I}\left(R^{*}\right)$ is uniformly bounded and equicontinuous on $I$.

Let $B \subset K \subset C[I, P]$ be any countable subset satisfying $\bar{B}=\overline{\mathrm{CO}}(\{u(t)\} \cap(F B)(t))$ for any $t \in I$. By applying Lemma 12 , we get

$$
\begin{gathered}
\Upsilon(B(t))=\overline{\Upsilon(B(t))}=\Upsilon((F B)(t)) \\
\leq 2 \int_{0}^{t}\left[\lambda_{1} \Upsilon(B(s))+\lambda_{2} \Upsilon((T B)(s))\right. \\
\left.+\lambda_{3} \Upsilon((S B)(s))\right] d s
\end{gathered}
$$

$$
\begin{aligned}
\leq & 2 \int_{0}^{t}\left[\lambda_{1} \Upsilon(B(s))+\lambda_{2} k_{0} \int_{0}^{s} \Upsilon(B(\tau)) d \tau\right. \\
& \left.\quad+\lambda_{3} h_{0} \int_{0}^{t_{0}+a} \Upsilon(B(\tau)) d \tau\right] d s \\
= & 2 \lambda_{1} \int_{0}^{t} \Upsilon(B(s)) d s \\
& +2 \lambda_{2} k_{0} \int_{0}^{t}(t-s) \Upsilon(B(s)) d s \\
& +2 \lambda_{3} h_{0} t \int_{0}^{t_{0}+a} \Upsilon(B(s)) d s \\
\leq & \left(2 \lambda_{1}+2 \lambda_{2} a k_{0}\right) \\
& \times \int_{0}^{t} \Upsilon(B(s)) d s+2 \lambda_{3} a h_{0} \int_{0}^{t_{0}+a} \Upsilon(B(s)) d s \\
= & M \int_{0}^{t} \Upsilon(B(s)) d s \\
& +M^{*} \int_{0}^{t_{0}+a} \Upsilon(B(s)) d s=0,
\end{aligned}
$$

where $M=\left(2 \lambda_{1}+2 \lambda_{2} a k_{0}\right), M^{*}=2 \lambda_{3} a h_{0}$. Thus, by AscoliArzela theorem, $B$ is relative compact in $X=C[I, E]$. It follows from Lemma 14 that $F$ has a fixed point $x^{*} \in K=$ $\overline{\mathrm{CO}}\left(F\left(B_{I}\left(R^{*}\right)\right)\right)$ which is a solution of IVP (1). This completes the proof of Theorem 18 .

Remark 19. The conditions imposed on nonlinear term do not possess any monotone properties. The results of the paper cannot be obtained by making use of the fixed point theorems on decreasing and increasing operators or mixed monotone operators.

\section{Conflict of Interests}

The author declares no conflict of interests.

\section{Acknowledgments}

The author is very grateful to Professor Lishan Liu, Professor Tijun Xiao, and Professor R. P. Agarwal for their many valuable comments. The work is supported financially by the Foundation of Shanghai Natural Science (13ZR1430100) and the Foundation of Shanghai Municipal Education Commission (DYL201105).

\section{References}

[1] D. Guo, "Extremal solutions of nonlinear Fredholm integral equations in ordered Banach spaces," Northeastern Mathematical Journal, vol. 7, no. 4, pp. 416-423, 1991.

[2] K. Deimling, Nonlinear Functional Analysis, Springer, New York, NY, USA, 1985.

[3] Y. B. Chen and W. Zhuang, "The existence of maximal and minimal solution of the nonlinear integro-differential equation 
in Banach space," Applicable Analysis, vol. 22, no. 2, pp. 139-147, 1986.

[4] Y. Du, "Fixed points of increasing operators in ordered Banach spaces and applications," Applicable Analysis, vol. 38, no. 1-2, pp. $1-20,1990$.

[5] S. Du and V. Lakshmikantham, "Monotone iterative technique for differential equations in a Banach space," Journal of Mathematical Analysis and Applications, vol. 87, no. 2, pp. 454-459, 1982.

[6] G. Ladde, V. Lakshmikantham, and A. Vatsala, Monotone Iterative Techniques for Nonlinear Differential Equations, Pitman, Boston, Mass, USA, 1985.

[7] V. Lakshmikantham and S. Leela, Nonlinear Differential Equations in Abstract Spaces, Pergamon Press, New York, NY, USA, 1981.

[8] V. Lakshmikantham, S. Leela, and A. Vatsala, "Method of quasi-upper and lower solutions in abstract cones," Nonlinear Analysis: Theory, Methods \& Applications, vol. 6, no. 8, pp. 833838, 1982.

[9] D. Guo, Semi-Ordered Method in Nonlinear Analysis, Shandong Science and Technology Press, Jinan, China, 2000 (Chinese).

[10] H. Su, L. Liu, and C. Wu, "Iterative solution for systems of a class of abstract operator equations and applications," Acta Mathematica Sinica, vol. 27, no. 3, pp. 449-455, 2007 (Chinese).

[11] X. Zhang, "Fixed point theorems for a class of nonlinear operators in Banach spaces and applications," Nonlinear Analysis: Theory, Methods \& Applications, vol. 69, no. 2, pp. 536-543, 2008.

[12] L. Liu, "Iterative method for solutions and coupled quasisolutions of nonlinear integro-differential equations of mixed type in Banach spaces," Nonlinear Analysis: Theory, Methods \& Applications, vol. 42, pp. 583-598, 2000.

[13] Y. Z. Chen, "Existence theorems of coupled fixed points," Journal of Mathematical Analysis and Applications, vol. 154, no. 1, pp. 142-150, 1991.

[14] D. Guo and V. Lakshmikantham, Nonlinear Problems in Abstract Cones, Academic Press, Boston, Mass, USA, 1988.

[15] T. G. Bhaskar and V. Lakshmikantham, "Fixed point theorems in partially ordered metric spaces and applications," Nonlinear Analysis: Theory, Methods \& Applications, vol. 65, no. 7, pp. 13791393, 2006.

[16] M. Krasnosel'skii and P. Zabreiko, Geometrical Methods of Nonlinear Analysis, Springer, Berlin, Germany, 1984.

[17] H. P. Heinz, "On the behaviour of measures of noncompactness with respect to differentiation and integration of vector-valued functions," Nonlinear Analysis: Theory, Methods \& Applications, vol. 7, no. 12, pp. 1351-1371, 1983.

[18] J. Banaś and K. Goebel, Measures of Noncompactness in Banach Spaces, vol. 60 of Lecture Notes in Pure and Applied Mathematics, Marcel Dekker, New York, NY, USA, 1980.

[19] H. Mönch, "Boundary value problems for nonlinear ordinary differential equations of second order in Banach spaces," Nonlinear Analysis: Theory, Methods \& Applications, vol. 4, no. 5, pp. 985-999, 1980.

[20] J. Sun and L. Liu, "Iterative method for coupled quasi-solutions of mixed monotone operator equations," Applied Mathematics and Computation, vol. 52, no. 2-3, pp. 301-308, 1992. 


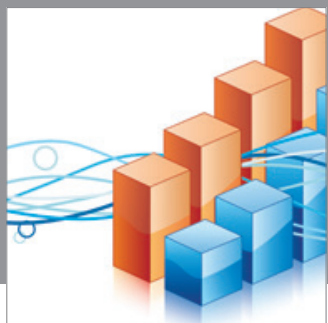

Advances in

Operations Research

mansans

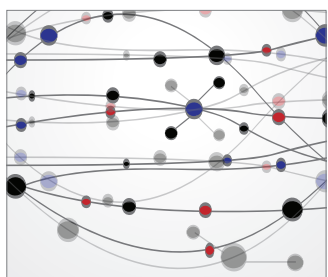

The Scientific World Journal
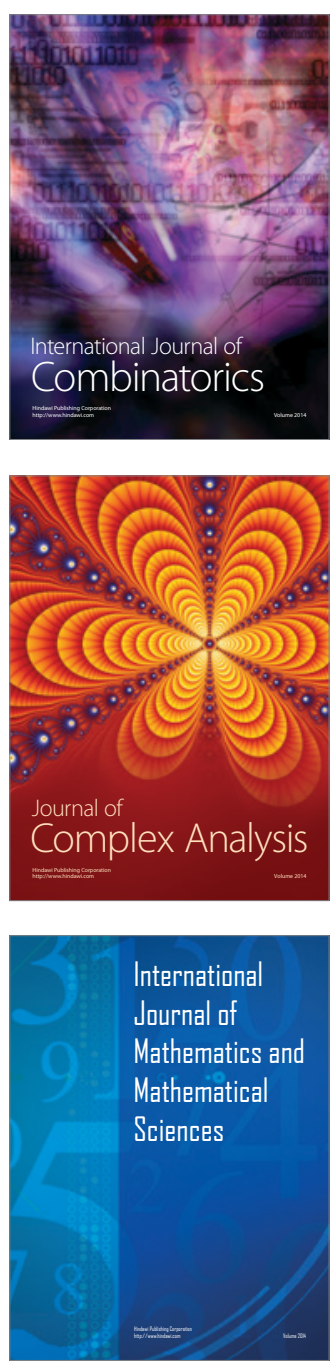
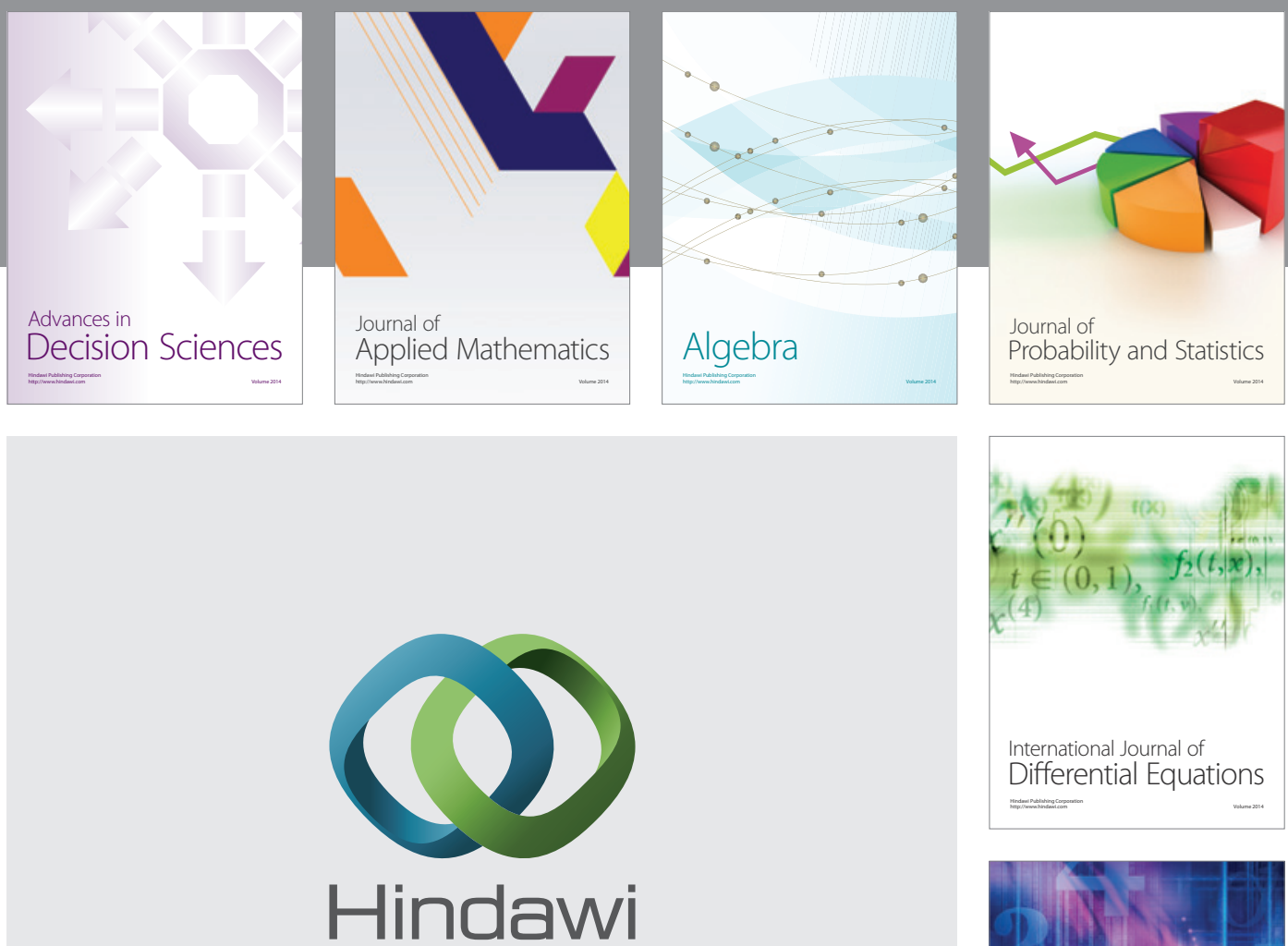

Submit your manuscripts at http://www.hindawi.com
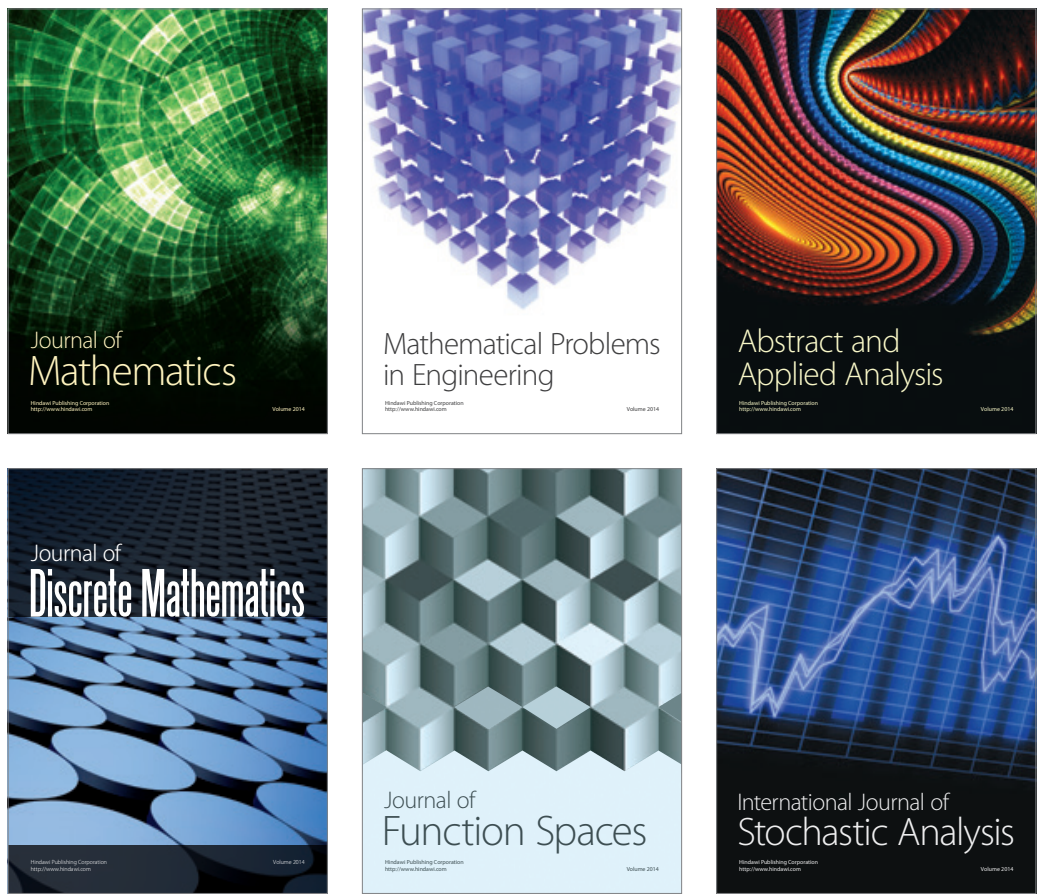

Journal of

Function Spaces

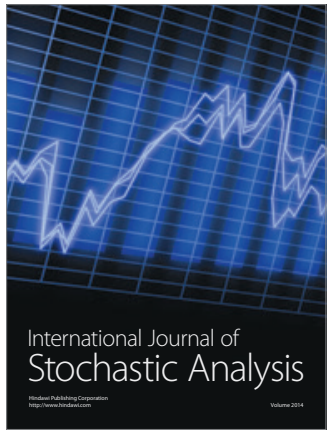

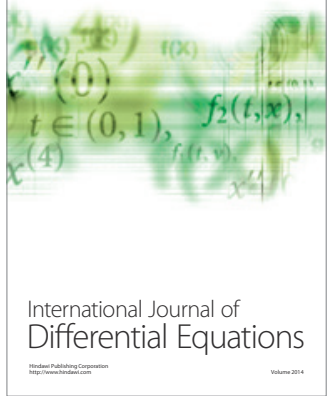
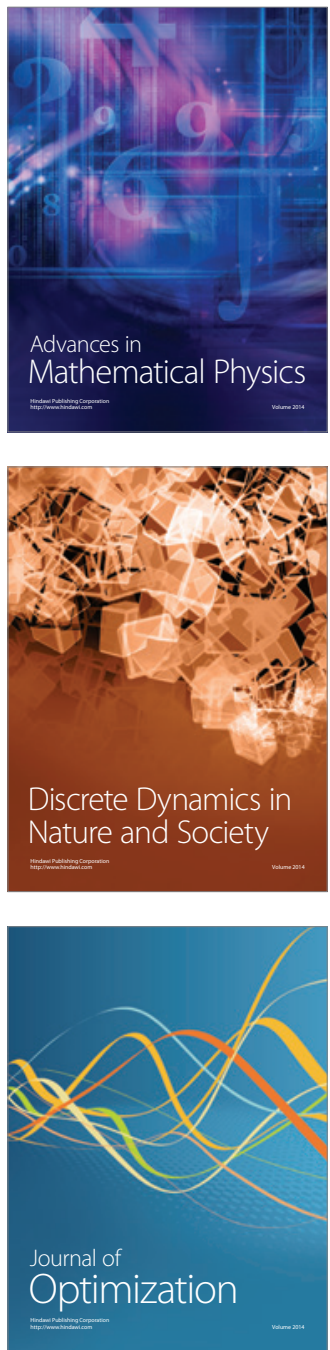